\title{
Ophiopogonin-D suppresses MDA-MB-435 cell adhesion and invasion by inhibiting matrix metalloproteinase-9
}

\author{
YUANYUAN ZHANG, YUWEI HAN, KEFENG ZHAI, MINHUI SUN, JIHUA LIU, \\ BOYANG YU and JUNPING KOU
}

\begin{abstract}
Department of Complex Prescription of Traditional Chinese Medicine, State Key Laboratory of Natural Medicines, China Pharmaceutical University, Nanjing, Jiangsu 211198, P.R. China
\end{abstract}

Received July 3, 2014; Accepted March 12, 2015

DOI: $10.3892 / \mathrm{mmr} .2015 .3541$

\begin{abstract}
Ophiopogonin-D is one of steroidal saponins isolated from the root of the Chinese medicinal plant Ophiopogon japonicas. It has been claimed to possess anti-inflammatory and anti-oxidant properties. The present study was the first to examine the anti-tumor metastasis properties of ophiopogonin-D. An MTT assay showed that ophiopogonin-D inhibited the proliferation of MDA-MB-435 melanoma cells, and decreased invasion was demonstrated using a Transwell invasion assay. Furthermore, adhesion of MDA-MB-435 cells to human umbilical vascular endothelial cells and to fibronectin was inhibited by ophiopogonin-D. Gelatin zymography and western blot analysis showed that ophiopogonin-D inhibited the expression and secretion of matrix metalloproteinase-9 (MMP-9), but not that of MMP-2. Inhibition of phosphorylation of p38 by ophiopogonin-D indicated its inhibition of the mitogen-activated protein kinase pathway. Overall, the results suggested that ophiopogonin-D may be considered as a candidate drug for treating or preventing tumor metastasis.
\end{abstract}

\section{Introduction}

Malignant neoplastic disease is one of the most common causes of mortality worldwide. $290 \%$ of all cancer mortalities are the result of metastases, rather than of the primary tumors (1). The major risk factors for malignant melanoma are personal or family history of melanoma, exposure to intense and intermittent ultraviolet irradiation, phenotypic characteristics, and multiple nevi (2). The process of tumor metastasis involves tumor cell proliferation, expansion, reorganization, degradation and migration through the surrounding stroma's

Correspondence to: Professor Junping Kou, Department of Complex Prescription of Traditional Chinese Medicine, State Key Laboratory of Natural Medicines, China Pharmaceutical University, 639 Longmian Road, Nanjing, Jiangsu 211198, P.R. China

E-mail: junpingkou@cpu.edu.cn

Key words: ophiopogonin-D, MDA-MB-435, invasion, adhesion microenvironment and the extracellular matrix (ECM), into the circulation to invade other tissues (3).

The process of tumor metastasis is accompanied with changes in gene expression, including mutation, overexpression, loss, activation or inactivation of numerous genes (4). The matrix metalloproteinase (MMP) family is a large group of secreted proteinases which require zinc for their catalytic activity. MMPs, which are proteolytic enzymes in the extracellular matrix (ECM), mainly contribute to cell motility, tumor invasion, angiogenesis and metastasis (5-7). Among these MMPs, MMP-2 (gelatinase-A) and MMP-9 (gelatinase-B) are mainly involved in cancer metastasis and invasion. They predominantly degrade gelatin and type IV, V, XI and XVI collagen, the major structural component of basement membrane, which appears to be crucial in tumor cell invasion and metastasis (8-10). A previous study reported that oroxylin A could inhibit migration and invasion of human breast MDA-MB-435 cancer cells via the inhibition of MMP-2 and MMP-9 (11). Thus, MMP-2 and MMP-9 have been considered as targets in the development of drugs against tumor invasion and metastasis $(12,13)$.

The mitogen-activated protein kinase (MAPK) pathway is associated with tumor proliferation and survival, motility and invasion (14). In melanoma, MAPK signalling cascades are critical and constitutively activated by a variety of mechanisms, making it a target for pathway targeting therapies. The MAPK signalling pathway is one of the most important cellular mechanisms responsible for melanoma metastasis by promoting cell proliferation, survival, invasion and tumor angiogenesis. Extracellular signal-regulated kinase 1 and 2 (ERK1/2) and p38/MAPK are major MAPKs, which are associated with tumor metastasis (15). They have a central role in regulating the expression of MMPs. The up- or downregulation of MAP kinases and their phosphorylation are involved in the regulation of MMP-9 expression in cancer cells $(16,17)$.

Medicinal plants have been used as traditional remedies for hundreds of years. Radix Ophiopogon japonicus is an important medicinal herb widely used for the treatment of various tumors, inflammatory diseases, hepatitis and diarrhea in East Asian countries, including China, Korea, Taiwan, and Japan (18-21). The plant has been reported to contain a large number of steroidal saponins. Ophiopogonin-D (ruscogenin $1-O-\alpha$-L-rhamnopyranosyl- $(1 \rightarrow 2)-\beta$-D-xylopyran osyl- ( $1 \rightarrow 3)-\beta$-D-fucopyranoside) (Fig. 1 ) is one of the steroidal 
saponins isolated from the root of Ophiopogon japonicus (22). According to previous studies, the ophiopogonin-D processes anti-inflammatory and anti-oxidant properties, including prevention of $\mathrm{H}_{2} \mathrm{O}_{2}$-induced injury in primary human umbilical vascular endothelial cells (HUVECs) (23), anti-thrombotic activity (24), anti-inflammation (25) and suppressed leukocyte migration in vivo (26). However, to the best of our knowledge, the anti-tumor activity of ophiopogonin-D has not been evaluated in vitro.

The present study investigated the molecular mechanisms by which ophiopogonin-D suppresses the migration and invasion of MDA-MB-435 cells in vitro. Furthermore, the underlying mechanism of the effect of ophiopogonin-D on MDA-MB-435 cells was explored by assessing its effect on MMP activity and the MAPK pathway. The present study provided evidence that ophiopogonin-D is suitable for use in the treatment of metastasis of melanoma.

\section{Materials and methods}

Chemicals and reagents. Ophiopogonin-D was isolated from the root of Ophiopogon japonicus according to the procedure of a previous study (27). Compounds were dissolved in dimethyl sulfoxide (DMSO; Nanjing Sunshine Biotechnology Ltd., Nanjing, China) and diluted with Dulbecco's medium Essential medium (DMEM; Invitrogen Life Technologies, Carlsbad, CA, USA) without serum prior to each experiment. The final concentration of DMSO in the culture medium never exceeded $0.1 \%$ (v/v), a concentration known not to affect cell proliferation, and control groups were always treated with $0.1 \%$ DMSO in the corresponding experiments. MTT, Rose Bengal and Triton X-100 were purchased from Sigma-Aldrich (St. Louis, MO, USA). High-glucose DMEM was purchased from Gibco-BRL (Invitrogen Life Technologies). Antibodies specific for phosphorylated (p-)ERK and p-p38/MAPK or total ERK, p38/MAPK, $\beta$-actin, MMP-2 and MMP-9 were obtained from Cell Signaling Technology (Cell Signaling Technology, Beverly, MA, USA). The cell culture plate and Transwell plate $(8-\mu \mathrm{m}$ pore size, $6.5-\mathrm{mm}$ diameter) were obtained from Corning Costar Corporation (Corning, NY, USA). Matrigel was purchased from BD Biosciences ( $\mathrm{La}$ Jolla, CA, USA).

Cells lines and culture. The human breast carcinoma cell line MDA-MB-435 was obtained from Keygene Corporation (Nanjing, China), grown in high-glucose DMEM supplemented with $10 \%$ heat-inactivated fetal bovine serum, $100 \mathrm{U} / \mathrm{ml}$ penicillin, $100 \mathrm{U} / \mathrm{ml}$ streptomycin and $3.7 \mathrm{~g} / 1$ sodium bicarbonate (all purchased from Nanjing Sunshine Biotechnology Ltd.). HUVECs were obtained from Shanghai FuMeng Gene Biotechnology Co., Ltd. (Shanghai, China). The cells were maintained under a humidified atmosphere of $95 \%$ air and $5 \%$ $\mathrm{CO}_{2}$ at $37^{\circ} \mathrm{C}$ and used for experiments whilst in logarithmic phase.

Cell adhesion of HUVECs and fibronectin. The tumor cell adhesion with HUVECs was detected according to the method by Jones et al (28) with certain modifications. HUVECs were seeded in 96-well plates in complete HUVEC medium at a density of $10^{4}$ cells per well for $24 \mathrm{~h}$. The HUVEC medium

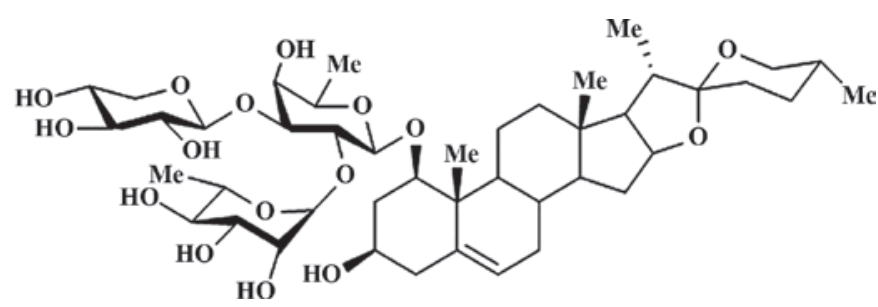

Figure 1. Chemical structure of ophiopogonin-D.

was then removed and cells were washed with phosphate-buffered saline (PBS). MDA-MB-435 cells were trypsinized and suspended at a final concentration of $5 \times 10^{5}$ cells $/ \mathrm{ml}$ with various concentrations of ophiopogonin-D (5, 10, 20, 40 and $80 \mu \mathrm{M}$ ) dissolved in serum-free DMEM. After $3 \mathrm{~h}$, the medium was removed and the undetached cells were washed with PBS twice and incubated with Rose Bengal for $5 \mathrm{~min}$. The cells were washed with PBS twice and fixed with $100 \mu \mathrm{l}$ mixed solution (95\% ethanol/PBS 1:1) per well for $30 \mathrm{~min}$ at room temperature. The ratio of adhesion was detected by measuring the absorbance of Rose Bengal at a wavelength of $600 \mathrm{~nm}$ using an ELISA reader (Bio-Tek Instruments, Winooski, VT, USA).

Cell adhesion to fibronectin was assayed as described previously with a few alterations (29). Briefly, 96-well plates were coated with fibronectin (Merck, Whitehouse Station, NJ, USA) at $4^{\circ} \mathrm{C}$ overnight. MDA-MB-435 cells, which were trypsinized and suspended at a final concentration of $5 \times 10^{5}$ cells/ml in serum-free DMEM with various concentrations of ophiopogonin-D and cultured for $3 \mathrm{~h}$. The unattached cells were removed by discarding the media and washing with PBS twice. A total of $100 \mu \mathrm{l}$ MTT $(0.5 \mathrm{mg} / \mathrm{ml}$ in PBS $)$ was added to the wells and the cells were cultured for another $3 \mathrm{~h}$. Following discarding the MMT solution, DMSO was then added to the wells. After agitation for $10 \mathrm{~min}$ at room temperature, the cytotoxicity was determined by measuring the absorbance of the converted dye at a wavelength of $570 \mathrm{~nm}$ and a reference wavelength of $650 \mathrm{~nm}$ in an ELISA reader.

Cell invasion assay. The cell invasion assay was determined as described previously with a few modifications (30). Briefly, the Transwell membrane $(8 \mu \mathrm{m}$ pore size, $6.5 \mathrm{~mm}$ diameter; Corning Costar Corporation) was pre-coated with Matrigel. The MDA-MB-435 cells, which were trypsinized and suspended at a final concentration of $5 \times 10^{5}$ cells $/ \mathrm{ml}$ in serum-free DMEM with various concentrations of ophiopogonin-D, were added to the upper well, while $500 \mu \mathrm{l}$ DMEM containing 10\% FBS was added to the lower well. After $20 \mathrm{~h}$, assays were stopped by removal of the medium from the upper wells. The cells on the membrane were fixed with $90 \%$ ethanol and cells on the upper side of the membrane, which had not transgressed through the membrane, were subsequently wiped off using cotton buds. The cells on the lower side of the membrane were stained with $0.1 \%$ methyl violet. After $10 \mathrm{~min}$, the chamber was washed with PBS and images of the membrane were captured using a microscope (Axiovert40CFL; Carl Zeiss, Oberkochen, Germany). Finally, the chamber was extracted with $10 \%$ acetic acid, and the absorbance of the extract was measured at $570 \mathrm{~nm}$ in an ELISA reader. 
A

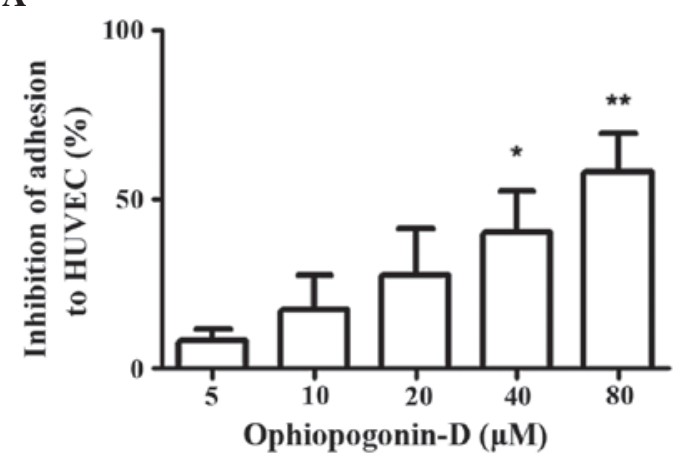

C

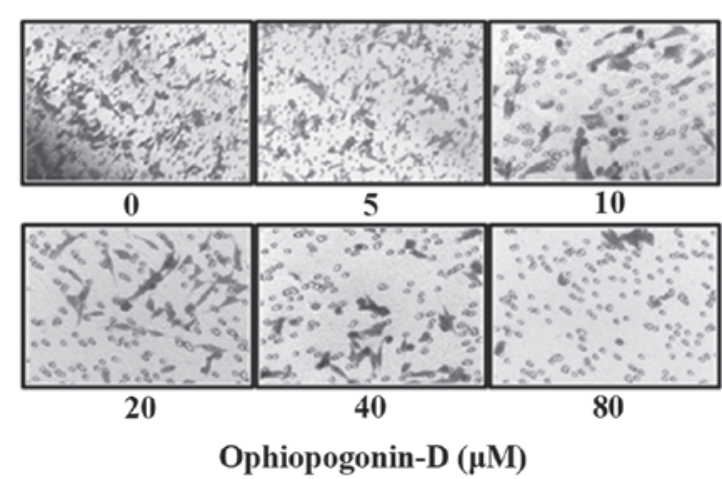

B
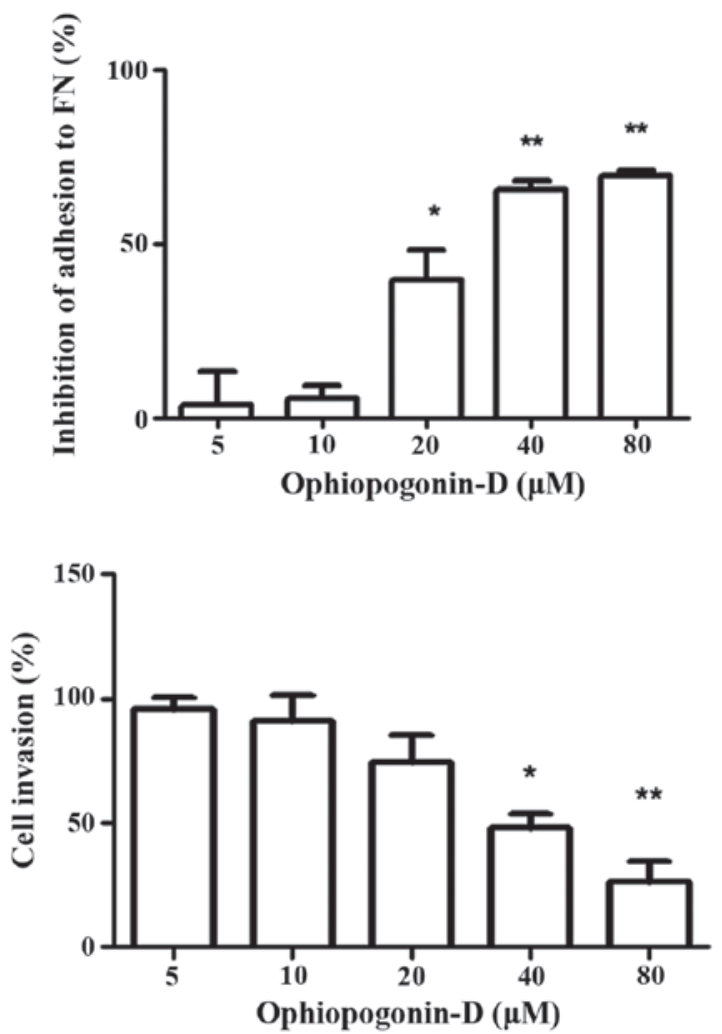

Figure 2. Effect of ophiopogonin-D on the adhesion and invasion of MDA-MB-435 cells. (A) Ophiopogonin-D inhibited the adhesion of MDA-MB-435 cells to HUVECs at 40 and $80 \mu \mathrm{M}$, as detected by Rose Bengal staining. (B) Ophiopogonin-D inhibited the adhesion of MDA-MB-435 cells to FN at 20, 40 and $80 \mu \mathrm{M}$, as detected by MTT assay. (C) Ophiopogonin-D inhibited the invasion of MDA-MB-435 cells in vitro as shown by a Transwell invasion assay. Values are expressed as the mean \pm standard deviation of three experiments (magnification, $\mathrm{x} 400$ ). ${ }^{*} \mathrm{P}<0.05,{ }^{* *} \mathrm{P}<0.01$ vs. the control group. FN, fibronectin; HUVEC, human umbilical vein endothelial cell.

Gelatin zymography. The activities of MMP-2 and MMP-9 were assayed by gelatin zymography $(31,32)$. Gelatin zymography was performed in $10 \%$ SDS-polyacrylamide gel containing $0.1 \%$ gelatin. After treatment with various concentrations of ophiopogonin-D $(5,10,20,40$ and $80 \mu \mathrm{M})$ for $24 \mathrm{~h}$, the supernatants were collected. Samples were mixed with loading buffer and electrophoresed. Gels were then washed twice in zymography washing buffer $(2.5 \%$ Triton X-100, $50 \mathrm{mmol} / \mathrm{l}$ Tris- $\mathrm{HCl}, 5 \mathrm{mmol} / \mathrm{l} \mathrm{CaCl}_{2}$; $\mathrm{pH}$ 7.6) for $45 \mathrm{~min}$ at room temperature to remove SDS, followed by rinsing twice in washing buffer for $20 \mathrm{~min}$. The gels were then incubated at $37^{\circ} \mathrm{C}$ for $40 \mathrm{~h}$ in zymography reaction buffer $(50 \mathrm{mmol} / \mathrm{l}$ Tris, $150 \mathrm{mmol} / 1 \mathrm{NaCl}, 10 \mathrm{mmol} / \mathrm{C} \mathrm{CaCl}_{2}, 0.02 \% \mathrm{NaN} 3, \mathrm{pH} 7.5$ ) and stained with $0.05 \%$ Coomassie blue R-250 (diluted in $30 \%$ methanol and $10 \%$ acetic acid) for $3 \mathrm{~h}$ and destained with destaining solution (10\% methanol and $5 \%$ acetic acid in $\mathrm{H}_{2} \mathrm{O}_{2}$ ). The field of the destained band was quantified using the Quantity One System (Bio-Rad, Hercules, CA, USA).

Western blot analysis. Activation of MMP-2/9 and MAPK were assessed by western blot analysis. MDA-MB-435 cells were pre-incubated with various concentrations of ophiopogonin-D for $20 \mathrm{~h}$, and cells were collected. Cells were then lysed in lysis buffer, and the equal amount of proteins was separated by SDS-PAGE and transferred onto the polyvinylidene difluoride membranes. Membranes were blocked with blocking buffer for $1.5 \mathrm{~h}$ at room temperature, followed by overnight incubation at $4^{\circ} \mathrm{C}$ in the relevant primary antibody $(1: 1,000)$, and finally blocked for $1 \mathrm{~h}$ with a secondary antibody HRP-conjugate $(1: 2,000)$. The band detection was revealed by enhanced chemiluminescence (ECL) using ECL western blotting detection reagents (Vazyme Biotech Co., Ltd., Nanjing, China) and exposure to ECL hyperfilm on Kodak film (Eastman Kodak, Rochester, NY, USA) and analyzed using the Quantity One System.

Statistical analysis. Values are expressed as the mean \pm standard deviation. The experimental data were analyzed using GraphPad Prism 5 software (GraphPad Inc., La Jolla, CA, USA). All comparisons were made relative to the control groups and significance of differences was indicated as ${ }^{*} \mathrm{P}<0.05$ and ${ }^{* *} \mathrm{P}<0.01$.

\section{Results}

Ophiopogonin-D inhibits MDA-MB-435 cell adhesion and invasion. In a preliminary experiment, the cytotoxic effects of ophiopogonin-D were determined using an MTT assay (data not shown). Ophiopogonin-D demonstrated no obvious cytotoxicity on MDA-MB-435 cells under $80 \mu \mathrm{M}$. Concentrations of ophiopogonin-D $(5,10,20,40$ and $80 \mu \mathrm{M})$ with no apparent cytotoxicity on MDA-MB-435 cells were selected for all subsequent experiments. As shown in Fig. 2A, ophiopogonin-D was able to inhibit the adhesion 


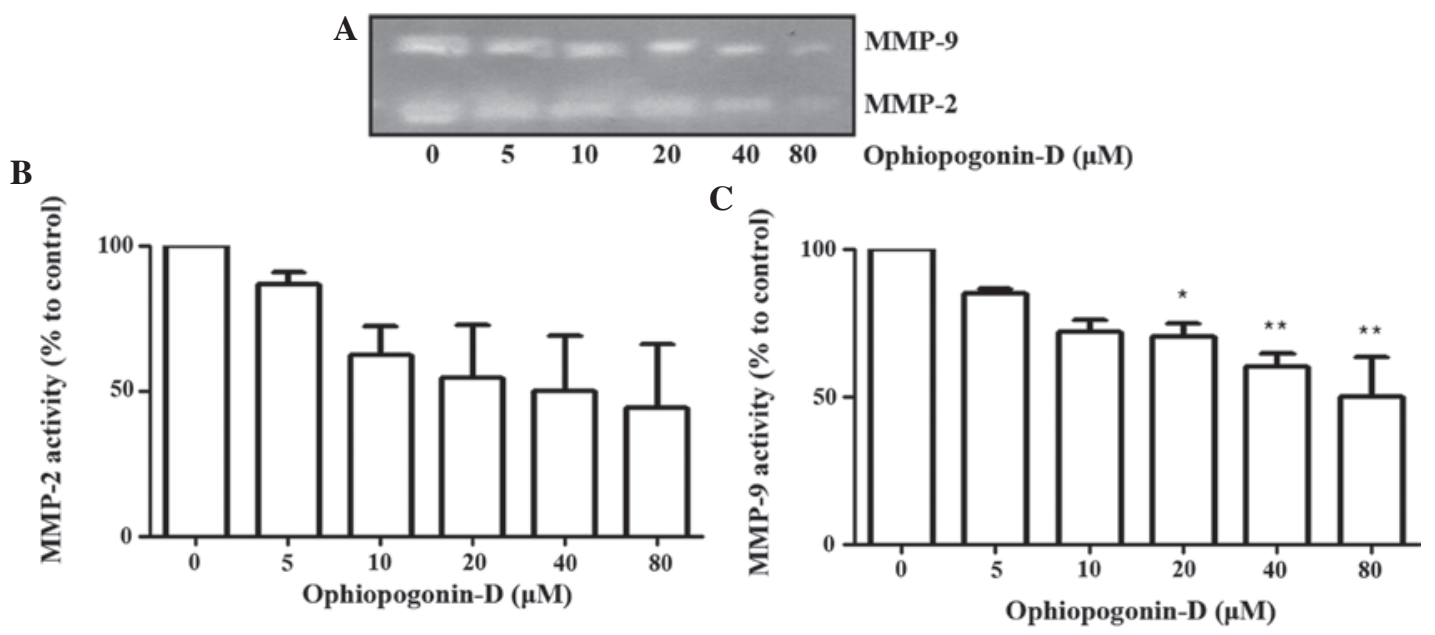

Figure 3. Effect of ophiopogonin-D on the activity of MMP-2/9 detected by (A) gelatin zymography. The relative enzyme activities of (B) MMP-2 and (C) -9 are expressed as percentages relative to control samples, quantified using the Quantity One System. " $\mathrm{P}<0.05$ and ${ }^{* * *} \mathrm{P}<0.01$ vs. the control. MMP, matrix metalloproteinase.
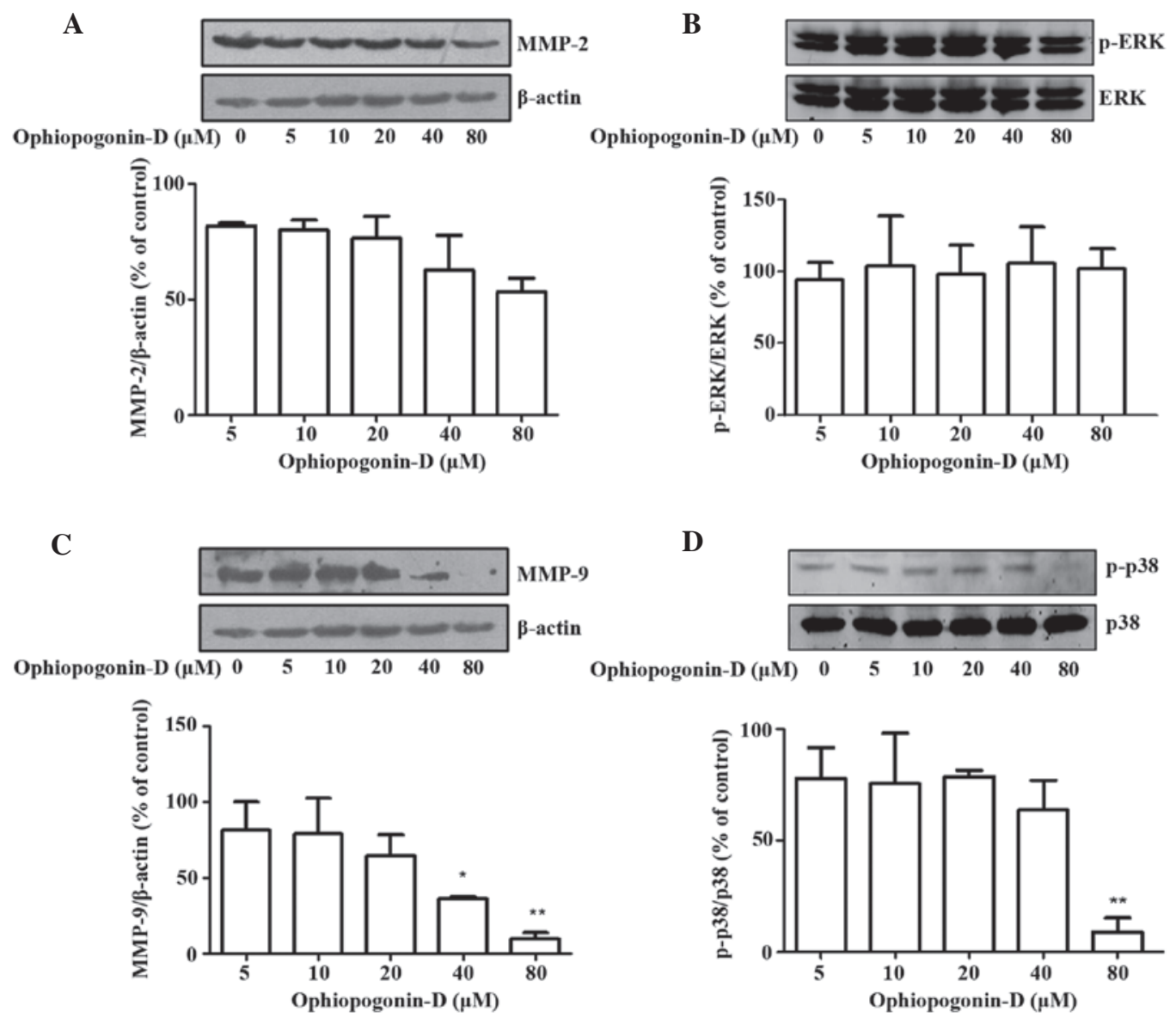

Figure 4. Effect of ophiopogonin-D on protein expression levels of (A) MMP-2, (B) p-ERk/ERK, (C) MMP-9 and (D) p-p38/p38 in MDA-M-435 cells as determined using western blot analysis. The intensity of the bands was densitometrically assessed. Values are expressed as the mean \pm standard deviation of three independent experiments. ${ }^{*} \mathrm{P}<0.05$ and ${ }^{* *} \mathrm{P}<0.01$, vs. control. MMP, matrix metalloproteinase; p-ERK, phosphorylated extracellular signal-regulated kinase.

of MDA-MB-435 cells to HUVECs. Ophiopogonin-D at concentrations of 40 and $80 \mu \mathrm{M}$ significantly reduced the number of MDA-MB-435 cells adhered to HUVECs, and the inhibition rates were $\sim 50$ and $65 \%$, respectively. In addition, MDA-MB-435-cell adhesion to fibronectin was detected for more accurate determination of the effect of ophiopogonin-D on the adhesion of melanoma cells. Fig. 2B shows the inhibitory effect of ophiopogonin-D on the adhesion of MDA-MB-435 cells to fibronectin. At concentrations of 40 and $80 \mu \mathrm{M}$, ophiopogonin-D significantly inhibited the adhesion of MDA-MB-435 cells to fibronectin at rates of $\sim 64$ and $80 \%$, respectively. Furthermore, the effect of ophiopogonin-D on the invasive ability of MDA-MB-435 cells was evaluated using a Transwell assay. Fig. $2 \mathrm{C}$ indicates that after treatment 
with ophiopogonin-D the invasive capacity of MDA-MB-435 cells was markedly inhibited. The inhibition rate of $80 \mu \mathrm{M}$ of ophiopogonin-D was $\sim 78 \%$.

Ophiopogonin-D decreases MMP-9, but not MMP-2 enzyme activity. The expression of MMP-2 and MMP-9 has been reported to have a critical role in degrading the basement membrane in tumor invasion and migration (33). A gelatin zymography assay showed that the activity of MMP-9 was reduced with increasing concentration of ophiopogonin-D (Fig. 3). MMP-9 was obviously decreased following incubation with 40 and $80 \mu \mathrm{M}$ ophiopogonin-D, while there was no obvious decrease in MMP-2. This result indicated that ophiopogonin-D mostly inhibits tumor metastasis through MMP-9, not MMP-2.

Effect of ophiopogonin-D on the expression of MMP-2/9 and MAPKs. MMPs have significant roles in cancer metastasis. Furthermore, the activity of MMP-2/9 is associated with the adhesion, invasion and angiogenesis of cancer metastasis $(34,35)$. Therefore, the effect of ophiopogonin-D on the expression of MMP-2 and MMP-9 was deteced by western blot analysis. The results indicated that ophiopogonin-D inhibited the expression of MMP-9 at concentrations of 40 and $80 \mu \mathrm{M}$ (Fig. 4). With increasing ophiopogonin-D concentration, the expression of MMP-9 declined, while there was no obvious decrease in MMP-2 expression. The result of the western blot analysis corresponded with the results of the adhesion and invasion experiments. Furthermore, the expression and phosphorylation of ERK1/2 and p38/MAPK were examined to assess whether the MAPK signaling pathway was involved in the anti-metastatic effect of ophiopogonin-D in melanoma cells (Fig. 4). The phosphorylation of p38 was inhibited by ophiopogonin-D at the concentration of $80 \mu \mathrm{M}$, while the phosphorylation of ERK was not inhibited by ophiopogonin-D. Further investigation is required to fully elucidate the underlying mechanism of the anti-metastatic effect of ophiopogonin-D on melanoma cells.

\section{Discussion}

Tumor growth, invasion and metastasis are multistep and complex processes that include cell division and proliferation, proteolytic digestion of the extracellular matrix, cell migration through basement membranes to reach the circulatory system, as well as re-migration and growth of tumors at the metastatic sites (36). The present study demonstrated that ophiopogonin-D, derived from the root of Ophiopogon japonicus, was able to inhibit the metastatic process in MDA-MB-435 cancer cells. It was found that ophiopogonin-D inhibited the proliferation, adhesion and invasion of MDA-MB-435 cells. Furthermore, the expression of MMP-9 and the activation of p38 were inhibited by ophiopogonin-D.

MMPs have a significant role in cancer metastasis (37). The activities of MMP-2 and MMP-9 are associated with the adhesion, invasion and angiogenesis of cancer metastasis $(38,39)$. Invasiveness inhibitors may act by downregulating extracellular barrier-degrading proteinases, including MMP-2 and/or MMP-9 (40). To further explore the mechanism of inhibition of invasion induced by ophiopogonin-D, the present study examined the effects of ophiopogonin-D on the expression and activity of MMP-2 and -9 in MDA-MB-435 human breast cancer cells. The results showed that ophiopogonin-D was able to inhibit the expression of MMP-9 and zymographic assays showed full development of the gelatinolytic potential of MMP-9 in the presence of invasion-restraining concentrations of ophiopogonin-D. It was demonstrated that ophiopogonin-D was able to concentration-dependently inhibit the secretion of MMP-9 in MDA-MB-435 cells. However, ophiopogonin-D had no obvious effects on the expression and secretion of MMP-2 in MDA-MB-435 cells. MMP-9, but not MMP-2, has an important role in the mechanism of the anti-tumor metastatic action of ophiopogonin-D. The results of the western blot analysis corresponded with the results of the adhesion and invasion assays.

To assess whether the MAPK signaling pathway is involved in the mechanism of action of ophiopogonin-D, the present study examined the expression and phosphorylation of ERK1/2 and p38 MAPK in ophiopogonin-D-treated cells. The expression of p-p38 was inhibited by ophiopogonin-D, but not the expression of p-ERK. The results indicated that ophiopogonin-D inhibited cell metastasis, likely through inactivation of $\mathrm{p} 38$, but not ERK1/2. It has been reported that a p38 pathway inhibitor was able to reduce MMP-9 expression and secretion, as well as in vitro invasion of cancer cells $(41,42)$. According to the results of the present study, ophiopogonin-D was able to reduce the expression and secretion of MMP-9 and the expression of p38/MAPK, and inhibit the invasion of MDA-MB- 435 cells. Therefore, it is concluded that ophiopogonin-D has the ability to act as a p38 inhibitor to impede tumor metastasis. Further investigation is required to fully elucidate the detailed mechanism of ophiopogonin-D on cancer metastasis, particularly with regard to the MAPK pathway and additional kinases.

In conclusion, the present study demonstrated that ophiopogonin-D, derived from the root of Ophiopogon japonicus, was able to inhibit the metastatic capacity of MDA-MB-435 cancer cells. Ophiopogonin-D effectively inhibited the proliferation, adhesion and invasion of MDA-MB-435 cells. Furthermore, the expression of MMP-9 was inhibited by ophiopogonin-D. The present study suggested that ophiopogonin-D inhibits tumor metastasis through downregulation of MMP-9 and suppression of the p38/MAPK pathway. Hence, ophiopogonin-D is suggested to be a therapeutic agent for inhibiting the metastasis and invasion of cancer.

\section{Acknowledgements}

The present study was supported by the National Natural Science Foundation of China (no. 81274131) and a General Financial Grant from the China Postdoctoral Science Foundation (no. 2012M521150).

\section{References}

1. Böhle AS and Kalthoff H: Molecular mechanisms of tumor metastasis and angiogenesis. Langenbecks Arch Surg 384: 133-140, 1999.

2. Miller AJ and Mihm MC Jr: Melanoma. N Engl J Med 355: 51-65, 2006.

3. Torzilli PA, Bourne JW, Cigler T and Vincent CT: A new paradigm for mechanobiological mechanisms in tumor metastasis. Semin Cancer Biol 22: 385-395, 2012. 
4. Perlikos F, Harrington KJ and Syrigos KN: Key molecular mechanisms in lung cancer invasion and metastasis: a comprehensive review. Crit Rev Oncol Hematol 87: 1-11, 2013.

5. Gao XH, Yang XQ, Wang BC, Liu SP and Wang FB: Overexpression of twist and matrix metalloproteinase-9 with metastasis and prognosis in gastric cancer. Asian Pac J Cancer Prev 14: 5055-5060, 2013.

6. Shuman Moss LA, Jensen-Taubman S and Stetler-Stevenson WG: Matrix metalloproteinases: changing roles in tumor progression and metastasis. Am J Pathol 181: 1895-1899, 2012

7. Bolkun L, Lemancewicz D, Sobolewski K, Mantur M, Semeniuk J, Kulczynska A, Kloczko J and Dzieciol J: The evaluation of angiogenesis and matrix metalloproteinase-2 secretion in bone marrow of multiple myeloma patients before and after the treatment. Adv Med Sci 58: 118-125, 2013.

8. Vandooren J, Van den Steen PE and Opdenakker G: Biochemistry and molecular biology of gelatinase B or matrix metalloproteinase-9 (MMP-9): the next decade. Crit Rev Biochem Mol Biol 48: 222-272, 2013

9. Monsonego-Ornan E, Kosonovsky J, Bar A, Roth L, Fraggi-Rankis V, Simsa S, Kohl A and Sela-Donenfeld D: Matrix metalloproteinase $9 /$ gelatinase B is required for neural crest cell migration. Dev Biol 364: 162-177, 2012.

10. Frankowski H, Gu YH, Heo JH, Milner R and Del Zoppo GJ: Use of gel zymography to examine matrix metalloproteinase (gelatinase) expression in brain tissue or in primary glial cultures. Methods Mol Biol 814: 221-233, 2012.

11. Sun Y, Lu N, Ling Y, Gao Y, Chen Y, Wang L, Hu R, Qi Q, Liu W, Yang Y, You Q and Guo Q: Oroxylin A suppresses invasion through down-regulating the expression of matrix metalloproteinase-2/9 in MDA-MB-435 human breast cancer cells. Eur J Pharmacol 603: 22-28, 2009.

12. Jin ML, Park SY, Kim YH, Park G and Lee SJ: Halofuginone induces the apoptosis of breast cancer cells and inhibits migration via downregulation of matrix metalloproteinase-9. Int J Oncol 44: 309-318, 2014

13. Liao CL, Lin JH, Lien JC, Hsu SC, Chueh FS, Yu CC, Wu PP, Huang YP, Lin JG and Chung JG: The crude extract of Corni Fructus inhibits the migration and invasion of U-2 OS human osteosarcoma cells through the inhibition of matrix metalloproteinase-2/-9 by MAPK signaling. Environ Toxicol 30: 53-63, 2015.

14. Cheng Y, Zhang G and Li G: Targeting MAPK pathway in melanoma therapy. Cancer Metastasis Rev 32: 567-584, 2013

15. Shen T, Heo SI and Wang MH: Involvement of the p38 MAPK and ERK signaling pathway in the anti-melanogenic effect of methyl 3,5-dicaffeoyl quinate in B16F10 mouse melanoma cells Chem Biol Interact 199: 106-111, 2012.

16. Davidson B, Givant-Horwitz V, Lazarovici P, Risberg B, Nesland JM, Trope CG, Schaefer E and Reich R: Matrix metalloproteinases (MMP), EMMPRIN (extracellular matrix metalloproteinase inducer) and mitogen-activated protein kinases (MAPK): co-expression in metastatic serous ovarian carcinoma. Clin Exp Metastasis 20: 621-631, 2003.

17. Wu KC, Yang ST, Hsia TC, Yang JS, Chiou SM, Lu CC, Wu RS and Chung JG: Suppression of cell invasion and migration by propofol are involved in down-regulating matrix metalloproteinase- 2 and p38 MAPK signaling in A549 human lung adenocarcinoma epithelial cells. Anticancer Res 32: 4833-4842, 2012.

18. Chen M, Du Y, Qui M, Wang M, Chen K, Huang Z, Jiang M, Xiong F, Chen J, Zhou J, Jiang F, et al: Ophiopogonin B-induced autophagy in non-small cell lung cancer cells via inhibition of the PI3K/Akt signaling pathway. Oncol Rep 29: 430-436, 2013.

19. Li N, Zhang L, Zeng KW, Zhou Y, Zhang JY, Che YY and Tu PF: Cytotoxic steroidal saponins from Ophiopogon japonicus. Steroids 78: 1-7, 2013.

20. Lan S, Yi F, Shuang L, Chenjie W and Zheng XW: Chemical constituents from the fibrous root of Ophiopogon japonicus and their effect on tube formation in human myocardial microvascular endothelial cells. Fitoterapia 85: 57-63, 2013.

21. Zhang Y, Liu J, Kou J, Yu J and Yu B: DT-13 suppresses MDA-MB-435 cell adhesion and invasion by inhibiting MMP-2/9 via the p38 MAPK pathway. Mol Med Rep 6: 1121-1125, 2012.

22. Yu BY, Qiu SX, Zaw K, Xu GJ, Hirai Y, Shoji J, Fong HH and Kinghorn AD: Steroidal glycosides from the subterranean parts of Liriope spicata var. prolifera. Phytochemistry 43: 201-206, 1996.

23. Qian J, Jiang F, Wang B, Yu Y, Zhang X, Yin Z and Liu C: Ophiopogonin D prevents $\mathrm{H} 2 \mathrm{O} 2$-induced injury in primary human umbilical vein endothelial cells. J Ethnopharmacol 128: $438-445,2010$
24. Kou J, Tian Y, Tang Y, Yan J and Yu B: Antithrombotic activities of aqueous extract from Radix Ophiopogon japonicus and its two constituents. Biol Pharm Bull 29: 1267-1270, 2006.

25. Kou J, Sun Y, Lin Y, Cheng Z, Zheng W, Yu B and Xu Q: Anti-inflammatory activities of aqueous extract from Radix Ophiopogon japonicus and its two constituents. Biol Pharm Bull 28: 1234-1238, 2005.

26. Huang YL, Kou JP, Ma L, Song JX and Yu BY: Possible mechanism of the anti-inflammatory activity of ruscogenin: role of intercellular adhesion molecule-1 and nuclear factor-kappaB. J Pharmacol Sci 108: 198-205, 2008.

27. Asano T, Murayama T, Hirai Y and Shoji J: Comparative studies on the constituents of ophiopogonis tuber and its congeners. VIII. Studies on the glycosides of the subterranean part of Ophiopogon japonicus Ker-Gawler cv. Nanus. Chem Pharm Bull (Tokyo) 41: 566-570, 1993.

28. Jones J, Marian D, Weich E, Engl T, Wedel S, Relja B, Jonas D and Blaheta RA: CXCR4 chemokine receptor engagement modifies integrin dependent adhesion of renal carcinoma cells Exp Cell Res 313: 4051-4065, 2007.

29. Lu ZJ, Ren YQ, Wang GP, Song Q, Li M, Jiang SS, Ning T, Guan YS, Yang JL and Luo F: Biological behaviors and proteomics analysis of hybrid cell line EAhy926 and its parent cell line A549. J Exp Clin Cancer Res 28: 16, 2009.

30. Chen HW, Lee JY, Huang JY, Wang CC, Chen WJ, Su SF Huang CW, Ho CC, Chen JJ, Tsai MF, Yu SL and Yang PC: Curcumin inhibits lung cancer cell invasion and metastasis through the tumor suppressor HLJ1. Cancer Res 68: 7428-7438, 2008.

31. Hung SH, Shen KH, Wu CH, Liu CL and Shih YW: Alpha-mangostin suppresses PC-3 human prostate carcinoma cell metastasis by inhibiting matrix metalloproteinase-2/9 and urokinase-plasminogen expression through the JNK signaling pathway. J Agric Food Chem 57: 1291-1298, 2009.

32. Kupai K, Szucs G, Cseh S, Hajdu I, Csonka C, Csont T and Ferdinandy P: Matrix metalloproteinase activity assays: Importance of zymography. J Pharmacol Toxicol Methods 61: 205-209, 2010

33. Hwang ES and Park KK: Magnolol suppresses metastasis via inhibition of invasion, migration and matrix metalloproteinase-2/-9 activities in PC-3 human prostate carcinoma cells. Biosci Biotechnol Biochem 74: 961-967, 2010.

34. Song C, Zhu S, Wu C and Kang J: Histone deacetylase (HDAC) 10 suppresses cervical cancer metastasis through inhibition of matrix metalloproteinase (MMP) 2 and 9 expression. J Biol Chem 288: 28021-28033, 2013.

35. Ho YL, Li KC, Chao W, Chang YS and Huang GJ: Korean red ginseng suppresses metastasis of human hepatoma SK-Hep1 cells by inhibiting matrix metalloproteinase-2/-9 and urokinase plasminogen activator. Evid Based Complement Alternat Med 2012: 965846, 2012.

36. Geho DH, Bandle RW, Clair T and Liotta LA: Physiological mechanisms of tumor-cell invasion and migration. Physiology (Bethesda) 20: 194-200, 2005.

37. Wu D, Huang P, Wang L, Zhou Y, Pan H and Qu P: MicroRNA-143 inhibits cell migration and invasion by targeting matrix metalloproteinase 13 in prostate cancer. Mol Med Rep 8: 626-630, 2013.

38. Khasigov PZ, Podobed OV, Gracheva TS, Salbiev KD, Grachev SV and Berezov TT: Role of matrix metalloproteinases and their inhibitors in tumor invasion and metastasis. Biochemistry (Mosc) 68: 711-717, 2003.

39. Hamsa TP and Kuttan G: Inhibition of invasion and experimental metastasis of murine melanoma cells by Ipomoea obscura (L) is mediated through the down-regulation of inflammatory mediators and matrix-metalloproteinases. J Exp Ther Oncol 9: 139-151, 2011.

40. Ordoñez R, Carbajo-Pescador S, Prieto-Dominguez N, García-Palomo A, González-Gallego J and Mauriz JL: Inhibition of matrix metalloproteinase- 9 and nuclear factor kappaB contribute to melatonin prevention of motility and invasiveness in HepG2 liver cancer cells. J Pineal Res 56: 20-30, 2013.

41. Yang JS, Lin CW, Hsieh YS, Cheng HL, Lue KH, Yang SF and Lu KH: Selaginella tamariscina (Beauv) possesses antimetastatic effects on human osteosarcoma cells by decreasing MMP-2 and MMP-9 secretions via p38 and Akt signaling pathways. Food Chem Toxicol 59: 801-807, 2013.

42. Khadjavi A, Valente E, Giribaldi G and Prato M: Involvement of p38 MAPK in haemozoin-dependent MMP-9 enhancement in human monocytes. Cell Biochem Funct 32: 5-15, 2014. 